\title{
Protein and vitamin B6 intake are associated with liver steatosis assessed by transient elastography, especially in obese individuals
}

\author{
Yvelise Ferro', Ilaria Carè', Elisa Mazza', Francesco Provenzano', Carmela Colica², Carlo Torti ${ }^{3}$, Stefano Romeo ${ }^{1,4}$, \\ Arturo Pujia', and Tiziana Montalcini ${ }^{5}$ \\ 'Department of Medical and Surgical Science, Nutrition Unit; ${ }^{2}$ Department of Pharmacology, Consiglio Nazionale Ricerche, Istituto \\ Scienze Neurologiche, University Magna Graecia; ${ }^{3}$ Department of Medical and Surgical Science, Unit of Infectious diseases, University \\ Magna Grecia, Catanzaro, Italy; ${ }^{4}$ Department of Molecular and Clinical Medicine, University of Gothenburg, Gothenburg, Sweden; \\ ${ }^{5}$ Department of Clinical and Experimental Medicine, Nutrition Unit, University Magna Grecia, Catanzaro, Italy
}

Background/Aims: Although the detrimental effects of several dietary components on the promotion of nonalcoholic fatty liver disease are well known, no studies have assessed the role of dietary vitamin B6. Moreover, studies on the associations between dietary components or body composition indices and liver steatosis assessed by transient elastography are rare. Our aim was to identify the nutritional factors and anthropometric parameters associated with liver steatosis.

Methods: In this cross-sectional study, we enrolled 168 individuals (35\% obese) who underwent a liver steatosis assessment by Controlled Attenuation Parameter measurement and nutritional assessment.

Results: Tertiles of vitamin $B 6$ intake were positively associated with hepatic steatosis ( $B=1.89, P=0.026$, confidence interval $[\mathrm{Cl}]$ 0.03-0.80) as well as with triglycerides, glucose, alanine aminotransferase (ALT), and body mass index . In obese individuals, after multivariable analysis, the Controlled Attenuation Parameter score was still associated with triglycerides, $\mathrm{ALT}$, and total protein intake ( $\mathrm{B}=0.56, P=0.01, \mathrm{Cl} 0.10-1.02)$. Participants in tertile I (low intake) had a lower Controlled Attenuation Parameter than those in tertile III $(P=0.01)$.

Conclusions: We found a positive association between hepatic steatosis or Controlled Attenuation Parameter score and vitamin B6/total protein intake, probably related to the high intake of meat. Vitamin B6 might have a pathogenic role related to the increase of hepatic steatosis. (Clin Mol Hepatol 2017;23:249-259)

Keywords: Obesity; Fatty liver; Elastography; Vitamin B6; Proteins

\section{INTRODUCTION}

Non-alcoholic fatty liver disease (NAFLD) is becoming the lead- ing cause of liver damage in Western countries.' Although the predisposition to progressive NAFLD is influenced by genetic heritability, ${ }^{2}$ the factors leading to the progression from simple liver

\section{Abbreviations:}

$\mathrm{AA}$, arachidonic acid; BMI, body mass index; $\mathrm{CAP}$, controlled attenuation parameter; FM, fat mass; $H D L$, high density lipoprotein; LDL, low density lipoprotein; NAFLD, Non-alcoholic fatty liver disease; TE, transient elastography

\section{Corresponding author : Tiziana Montalcini}

Department of Clinical and Experimental Medicine, Nutrition Unit, University Magna Grecia of Catanzaro, Viale Europa, 88100 Catanzaro, Italy Tel: +39-0961-3695172, Fax: +39-0961-3694520

E-mail: tmontalcini@unicz.it

http://orcid.org/0000-0001-7048-5830 
steatosis to severe liver scarring and cirrhosis are not fully clear. One of the key mediators of this process may be arachidonic acid $(\mathrm{AA}){ }^{3}$ which is a potent pro-inflammatory molecule. ${ }^{4,5}$ The plasma AA level and its liver content depend on vitamin B6 bioavailability. ${ }^{5-7}$ It also been demonstrated that highest vitamin B6 concentration stimulates AA-derived eicosanoid production in the liver. ${ }^{8}$ However, to date, human studies investigating the association between dietary vitamin B6 and liver steatosis are lacking.

Recently, transient elastography (TE) was developed to detect and quantify liver steatosis by means of a non-invasive parameter, i.e. the controlled attenuation parameter (CAP) which correlates with the histopathological evaluation of hepatic steatosis. ${ }^{9,10}$ Epidemiological data on the association between dietary components or body composition indices and hepatic steatosis assessed with this new test are very limited. Consequently, the primary aim of this study was to find out which nutritional factors, including vitamin B6, and body composition parameters were associated with the presence of hepatic steatosis. The primary outcome measure was hepatic fat content quantified using the CAP score. This study also aimed to investigate whether there was a difference in the CAP score depending on the different ranges of vitamin B6 intake.

\section{MATERIALS AND METHODS}

In this cross-sectional study we enrolled adult individuals invited by newspaper advertisements to be screened for the possible presence of liver steatosis by TE. The investigation was conducted from July to December 2015 at the "Mater Domini" Azienda University Hospital. The protocol was approved by local ethical committee of this Hospital (protocol number 2013.11). Written informed consent was obtained from all participants. The investigation conforms to the principles outlined in the Declaration of Helsinki.

\section{Exclusion criteria}

During the medical interview and examination, we excluded from the study those who, had clinical and laboratory signs of chronic hepatitis $B$ and/or $C$ virus infection, past and current alcohol abuse (> $20 \mathrm{~g}$ of alcohol per day; $350 \mathrm{~mL}$ (12 oz) of beer, 120 $\mathrm{mL}(4 \mathrm{oz})$ of wine, and $45 \mathrm{~mL}(1.5 \mathrm{oz}$ ) of hard liquor each contain $10 \mathrm{~g}$ of alcohol), current use or history of drug treatment causing hepatic steatosis, impaired liver function, presence of autoimmune or cholestatic liver disease, Type 1 and 2 diabetes mellitus, and those to whom it was not possible to measure CAP score for technical reasons. The following criteria were used to define diabetes: fasting blood glucose $\geq 126 \mathrm{mg} / \mathrm{dL}$ or antidiabetic treatment.

\section{Liver transient elastography}

TE can quantify liver steatosis by CAP assessment and measure liver stiffness. ${ }^{12}$ (Fibroscan ${ }^{\circledR}$; Echosense SASU, Paris, France). Both stiffness and CAP score were obtained simultaneously and in the same volume of liver parenchyma. All patients were evaluated using the $3.5 \mathrm{MHz}$ standard $\mathrm{M}$ probe on the right lobe of the liver through intercostal spaces with the patient lying supine and placing the right arm behind the head to facilitate access to the right upper quadrant of the abdomen. The tip of the probe transducer was placed on the skin between the rib bones at the level of the right lobe of the liver. All scans were performed by the same investigator. Liver stiffness was expressed by the median value (in $\mathrm{kPa}$ ) of ten measurements performed between 25 and $65 \mathrm{~mm}$ depth. Only results with 10 valid shots and interquartile range $(I Q R) /$ median liver stiffness ratio $<30 \%$ were included. The cut-off value for defining the presence of fibrosis was liver stiffness $>7$ $\mathrm{kPa}$.

We assessed CAP score using only the M probe because the CAP algorithm is specific to this device. Ten successful measurements were performed on each patient, and only cases with ten successful acquisitions were taken into account for this study. The success rate was calculated as the number of successful measurements divided by the total number of measurements. The ratio of the IQR of liver stiffness to the median (IQR/MLSM) was calculated as an indicator of variability. The final CAP score (ranged from 100 to 400 decibels per meter $(\mathrm{dBm}-)$, was the median of individual measurements. The ratio of IQR in CAP values to the median (IQR/ $M(A P)$ was used as an indicator of variability for the final $C A P^{9}$. In order to identify the presence of hepatic steatosis, three different cut-offs were evaluated: 216,250 and $300 \mathrm{~dB} / \mathrm{m}^{10,11}$

\section{Dietary intake and cardiovascular risk factors assessment}

Dietary intake data were assessed by a 24-hour recall and a seven-day food record, ${ }^{12,13}$ and calculated using nutritional software MetaDieta 3.0.1 (Metedasrl, San Benedetto del Tronto, Italy). Precisely, the 24-hour recall was performed via a face-to-face interview with a dietitian who used images associated with a 
comprehensive food list. The recall required 15-20 min to complete for each participant. The patients were also asked to report any ingredients, food and food waste in a food diary for a 7-day period. Each patient was trained by a skilled dietitian before starting the study. The dietitian showed how various foods should be recorded. The portion sizes used were based on the typical or natural portion consumed (e.g., a slice of bread, one egg). When a typical portion size was not obvious, a commonly-used portion size was selected (e.g., one cup). The nutrient database used to calculate nutrient intake was derived primarily from Istituto Nazionale Ricerca Alimentazione e Nutrizione (Italian National Research Institute for Food and Nutrition, INRAN) 2000 and European Institute of Oncology (IEO) 2008. ${ }^{13}$ This database includes over 5,000 foods and brand name products, and is updated annually.

Briefly, dietary intake data were entered directly into the software MetaDieta. The software searches for foods and brand products by name. The coding of foods and their variable ingredients occurs as data are entered, with the simultaneous immediate calculation of nutrients. Nutrient values and other food components were generated from the database together with food group assignments.

We assessed the presence of the classical cardiovascular (CV) risk factors, such as hyperlipidemia, hypertension, diabetes and smoking, from clinical records and patient interview. ${ }^{12,14,15}$ Blood pressure was determined at the time of the visit. Obesity was defined by the presence of a body mass index (BMI) $\geq 30 \mathrm{~kg} / \mathrm{m}^{2}$.

\section{Biochemical evaluation}

Venous blood was collected after fasting overnight into vacutainer tubes (Becton \& Dickinson, Plymouth, England) and centrifuged within $4 \mathrm{~h}$. Serum glucose, total cholesterol, high density lipoprotein (HDL)-cholesterol, triglycerides were measured with Enzymatic colorimetric test. Low-density lipoprotein (LDL) cholesterol level was calculated by the Friedewald formula. ${ }^{16}$ Quality control was assessed daily for all determinations.

\section{Anthropometric measurements}

Body weight was measured before breakfast with a calibrated scale and height measured with a wall-mounted stadiometer. BMI was calculated with the following equation: weight $(\mathrm{kg}) / \mathrm{height}$ $(\mathrm{m})^{2}$. Waist circumferences and hip circumferences (WC and HC) were measured with a nonstretchable tape. ${ }^{17}$ Bioelectrical impedance analysis (BIA) (BIA-101, Akern srl, Florence, Italy) was per- formed to estimate the percentage of Fat Mass (FM) ${ }^{17,18}$

\section{Statistical analysis}

Data were reported as mean \pm SD. A minimum of ninety eight subjects are required to detect a significant CAP score difference of at least $10 \%$ between vitamin B6 tertiles, with $80 \%$ power on a two-sided level of significance of 0.05 .

Pearson's correlation was used first in the whole population and it was, then, used in obese individuals to identify the variables correlated with CAP score given that the continuous variables were normally distributed. We analyzed the correlation with the following variables: age, BMI, WC, $H C$, glucose, $L D L, H D L$, triglycerides, ALT, AST, SBP, DBP, FM, intake of calories, carbohydrates, fat, total protein, animal-based protein, plant-based protein, animal fat, vegetal fat, monounsaturated and polyunsaturated fatty acids, cholesterol, saturated fat, vitamins, amino acids, alcohol per day.

We categorized the whole population and, subsequently, only those with obesity, according to tertiles of vitamin B6 intake. A chi square test was performed to compare the prevalence and ANOVA was performed to compare the means between tertiles, with a Fisher's LSD test as a post-hoc analysis.

A logistic regression analysis was performed to test the association between tertiles of vitamin B6 intake and all of the potentially confounding variables, as independent variables, with hepatic steatosis serving as the dependent variable (binary variable; CAP score $>300 \mathrm{~dB} / \mathrm{m})^{11}$ (potential confounders were all those factors correlated with CAP score in the univariate analysis with a $P$-value $<0.1$ ).

In addition, stepwise multivariable linear regression analysis was used in both the whole population and the obese. The main independent variable plus all of the confounding variables were entered into one model, with the CAP score as independent variables.

Finally, in those obese, a Pearson's correlation was used to identify the food groups eventual correlated with vitamin B6 intake, that were the following: grains, meat, alcohol, beer, seed oils, trans saturated fatty acid, cheeses and calories. Significant differences were assumed to be present at $P<0.05$ (two-tailed). All analyzes were performed using SPSS 20.0 for Windows (S. Wacker Drive, Chicago, Illinois 60606, USA).

\section{RESULTS}

A total of 168 subjects were consecutively enrolled in the study. 
Table 1. Participant demographic, anthropometric, and clinical characteristics (whole population)

\begin{tabular}{lc}
\hline Variables & Value \\
\hline Age (years) & $44(16)$ \\
\hline BMI (kg/m²) & $29(6)$ \\
\hline WC (cm) & $95(15)$ \\
HC (cm) & $108(12)$ \\
\hline SBP (mmHg) & $123(14)$ \\
\hline DBP (mmHg) & $76(9)$ \\
\hline FFM (\%) & $69(10)$ \\
\hline FM (\%) & $31(10)$ \\
\hline CAP score (dB/m) & $248(55)$ \\
\hline Liver Stiffness (kPa) & $4.9(1.3)$ \\
\hline Glucose (mg/dL) & $91(10)$ \\
\hline Total cholesterol (mg/dL) & $188(35)$ \\
\hline HDL cholesterol (mg/dL) & $56(15)$ \\
\hline LDL cholesterol (mg/dL) & $111(32)$ \\
\hline Triglycerides (mg/dL) & $107(71)$ \\
\hline AST (IU/L) & $21(8)$ \\
\hline ALT (IU/L) & $22(14)$ \\
\hline Prevalence (\%) & 24 \\
\hline Obesity & 74 \\
\hline Hyperlipidemia & 35 \\
\hline Hypertension & 23 \\
\hline Smokers & 24 \\
\hline
\end{tabular}

Values are presented as mean (SD) unless otherwise indicated.

$\mathrm{BMI}$, body mass index; WC, waist circumferences; $\mathrm{HC}$, hip circumferences; SBP, systolic blood pressure; DBP, diastolic blood pressure; FFM, free fat mass; FM, fat mass; CAP, controlled attenuation parameter; HDL, high density lipoprotein; LDL, low density lipoprotein; AST, aspartate aminotransferase; ALT, alanine aminotransferase.

Table 1 shows the participants' demographic, anthropometrics and clinical characteristics. Mean age was $44 \pm 16$ years. Mean BMI was $29 \pm 6 \mathrm{~kg} / \mathrm{m}^{2}$. A total of 58 individuals (34.5\%) were obese. In the whole population, $75 \%$ had a CAP value $\geq 216$, $47 \% \geq 250$, and $20 \% \geq 300 \mathrm{dBm}-1$.

Table 2 shows the factors correlated with the CAP score. Vitamin B6 correlated with the CAP score $(r=0.13 ; P=0.08)$. As expected, BMI, WC, glucose, triglycerides, ALT, AST correlated with the CAP score $(\mathrm{r}=0.51, P=0.001 ; \mathrm{r}=0.60, P<0.001 ; \mathrm{r}=0.35$, $P<0.001 ; \mathrm{r}=0.44, P<0.001 ; \mathrm{r}=0.32, P<0.001 ; \mathrm{r}=0.46, P<0.001$, respectively) while no association was found with alcohol or beer consumption. Furthermore, total carbohydrates, fat and protein intake and monounsaturated fatty acids and saturated fat corre-

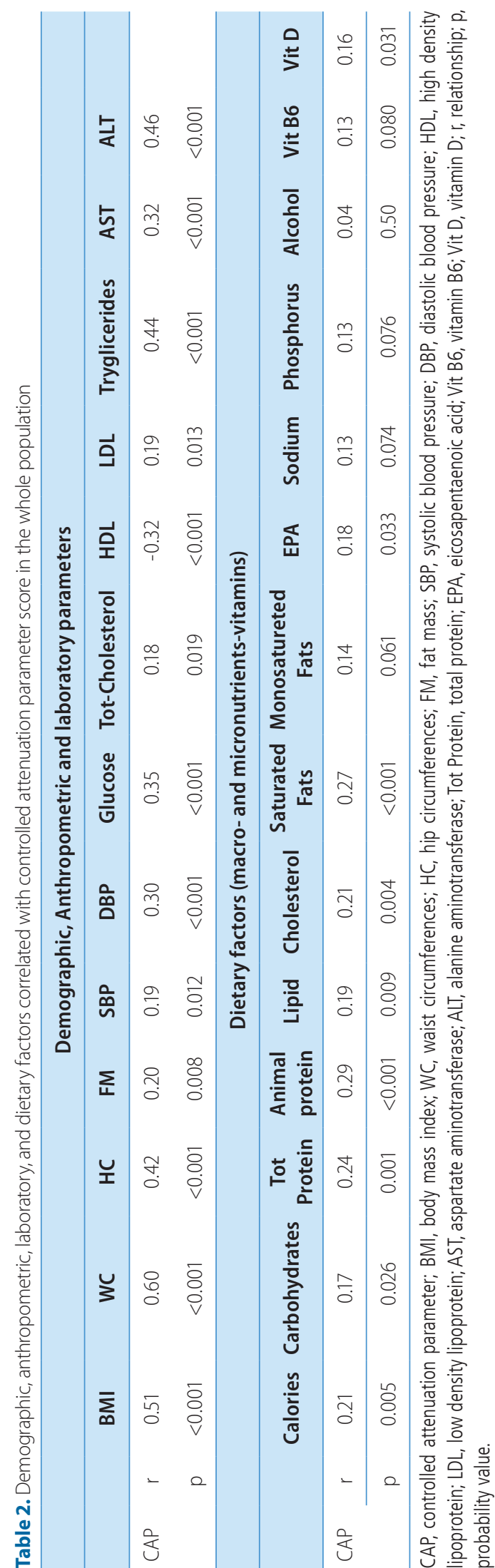


Yvelise Ferro, et al.

Liver and vitamin B6

Table 3. Participant demographic, anthropometric, and clinical characteristics according to vitamin B6 tertile of intake (whole population)

\begin{tabular}{|c|c|c|c|c|c|}
\hline Variables & $\begin{array}{c}\text { I tertile }(n=56) \\
1.13 \pm 0.2 \mathrm{mg} / \text { day }\end{array}$ & $\begin{array}{c}\text { Il tertile }(n=56) \\
1.66 \pm 0.2 \mathrm{mg} / \text { day }\end{array}$ & $\begin{array}{l}\text { III tertile }(n=56) \\
2.41 \pm 0.6 \mathrm{mg} / \text { day }\end{array}$ & $P$-value & $\begin{array}{c}\text { P-value } \\
\text { Post-Hoc Analysis }\end{array}$ \\
\hline Age (years) & $46 \pm 16$ & $43 \pm 17$ & $43 \pm 16$ & 0.47 & NS \\
\hline $\mathrm{BMI}\left(\mathrm{kg} / \mathrm{m}^{2}\right)$ & $28.5 \pm 6$ & $28.3 \pm 7$ & $30.2 \pm 6$ & 0.16 & NS \\
\hline WC (cm) & $94 \pm 15$ & $93 \pm 15$ & $98 \pm 16$ & 0.12 & NS \\
\hline $\mathrm{HC}(\mathrm{cm})$ & $107 \pm 10$ & $106 \pm 13$ & $110 \pm 12$ & 0.16 & NS \\
\hline $\mathrm{SBP}(\mathrm{mmHg})$ & $121 \pm 12$ & $121 \pm 13$ & $125 \pm 17$ & 0.23 & NS \\
\hline $\mathrm{DBP}(\mathrm{mmHg})$ & $75 \pm 9$ & $75 \pm 9$ & $78 \pm 9$ & 0.13 & NS \\
\hline FFM (\%) & $68 \pm 10$ & $69 \pm 11$ & $69 \pm 9$ & 0.68 & NS \\
\hline FM (\%) & $32 \pm 10$ & $31 \pm 11$ & $31 \pm 9$ & 0.68 & NS \\
\hline CAP score (dB/m) & $234 \pm 54$ & $249 \pm 58$ & $263 \pm 48$ & 0.017 & | vs. ||| 0.004 \\
\hline Liver stiffness (kPa) & $4.9 \pm 1.3$ & $4.7 \pm 1.2$ & $5.2 \pm 1.3$ & 0.17 & NS \\
\hline Glucose (mg/dL) & $89 \pm 10$ & $91 \pm 10$ & $92 \pm 11$ & 0.28 & NS \\
\hline Total cholesterol (mg/dL) & $184 \pm 32$ & $188 \pm 37$ & $194 \pm 35$ & 0.35 & NS \\
\hline HDL cholesterol (mg/dL) & $57 \pm 16$ & $55 \pm 15$ & $56 \pm 15$ & 0.78 & NS \\
\hline LDL cholesterol (mg/dL) & $108 \pm 30$ & $111 \pm 30$ & $116 \pm 34$ & 0.44 & NS \\
\hline Triglycerides (mg/dL) & $95 \pm 51$ & $107 \pm 63$ & $120 \pm 93$ & 0.18 & NS \\
\hline AST (IU/L) & $21 \pm 9$ & $22 \pm 9$ & $20 \pm 7$ & 0.53 & NS \\
\hline ALT (IU/L) & $20 \pm 13$ & $24 \pm 16$ & $23 \pm 14$ & 0.37 & NS \\
\hline \multicolumn{6}{|l|}{ Prevalence } \\
\hline Hyperlipidemia (\%) & 24 & 27 & 17 & 0.40 & \\
\hline Hypertension (\%) & 29 & 25 & 17 & 0.29 & \\
\hline Smokers (\%) & 75 & 71 & 78 & 0.77 & \\
\hline
\end{tabular}

BMI, body mass index; WC, waist circumferences; HC, hip circumferences; SBP, systolic blood pressure; DBP, diastolic blood pressure; FFM, free fat mass; FM, fat mass; CAP, controlled attenuation parameter; HDL, high density lipoprotein; LDL, low density lipoprotein; AST, aspartate aminotransferase; ALT, alanine aminotransferase; NS, non significant.

Table 4. Factors associated with hepatic steatosis in the whole population in logistic regression analysis

\begin{tabular}{lcccc}
\hline Dependent variable (Hepatic steatosis) & B & SE & p & OR (C.I. 95\%) \\
\hline BMI & 0.26 & 0.11 & 0.023 & $1.29(1.04-1.61)$ \\
Glucose & 0.15 & 0.06 & 0.009 & $1.16(1.04-1.30)$ \\
Triglycerides & 0.02 & 0.01 & 0.004 & $1.02(1.01-1.04)$ \\
ALT & 0.14 & 0.04 & 0.001 & $1.15(1.06-1.25)$ \\
Tertiles of Vitamin B6 intake & -1.89 & 0.85 & 0.026 & $0.15(0.03-0.80)$ \\
\hline
\end{tabular}

B, unstandardized coefficients; SE, standard errors; p, probability value; OR, odds ratio; C.I., confidence interval; BMI, body mass index; ALT, alanine aminotransferase.

Table 5. Factors associated with controlled attenuation parameter score in the whole population in multivariable linear regression analysis

\begin{tabular}{|c|c|c|c|c|c|c|}
\hline \multirow{2}{*}{ Dependent variable (CAP score) } & \multirow{2}{*}{ B } & \multirow{2}{*}{ SE } & \multirow{2}{*}{$\beta$} & \multirow{2}{*}{$\mathrm{p}$} & \multicolumn{2}{|c|}{ C.I. $95 \%$} \\
\hline & & & & & LL & UL \\
\hline BMl & 2.64 & 0.61 & 0.29 & $<0.001$ & 1.4 & 3.9 \\
\hline Glucose & 0.72 & 0.36 & 0.13 & 0.048 & 0.08 & 1.4 \\
\hline Triglycerides & 0.99 & 0.05 & 0.24 & $<0.001$ & 0.08 & 0.27 \\
\hline ALT & 1.05 & 0.5 & 0.24 & $<0.001$ & 0.53 & 1.5 \\
\hline Tertiles of Vitamin B6 intake & 8.14 & 4.0 & 0.12 & 0.044 & 0.01 & 16 \\
\hline
\end{tabular}

CAP, controlled attenuation parameter; B, unstandardized coefficients; SE, standard errors; $\beta$, standardized coefficients; $p$, probability value; C.I., confidence interval; LL, lower limit; UL, upper limit; BMI, body mass index; ALT, alanine aminotransferase. 


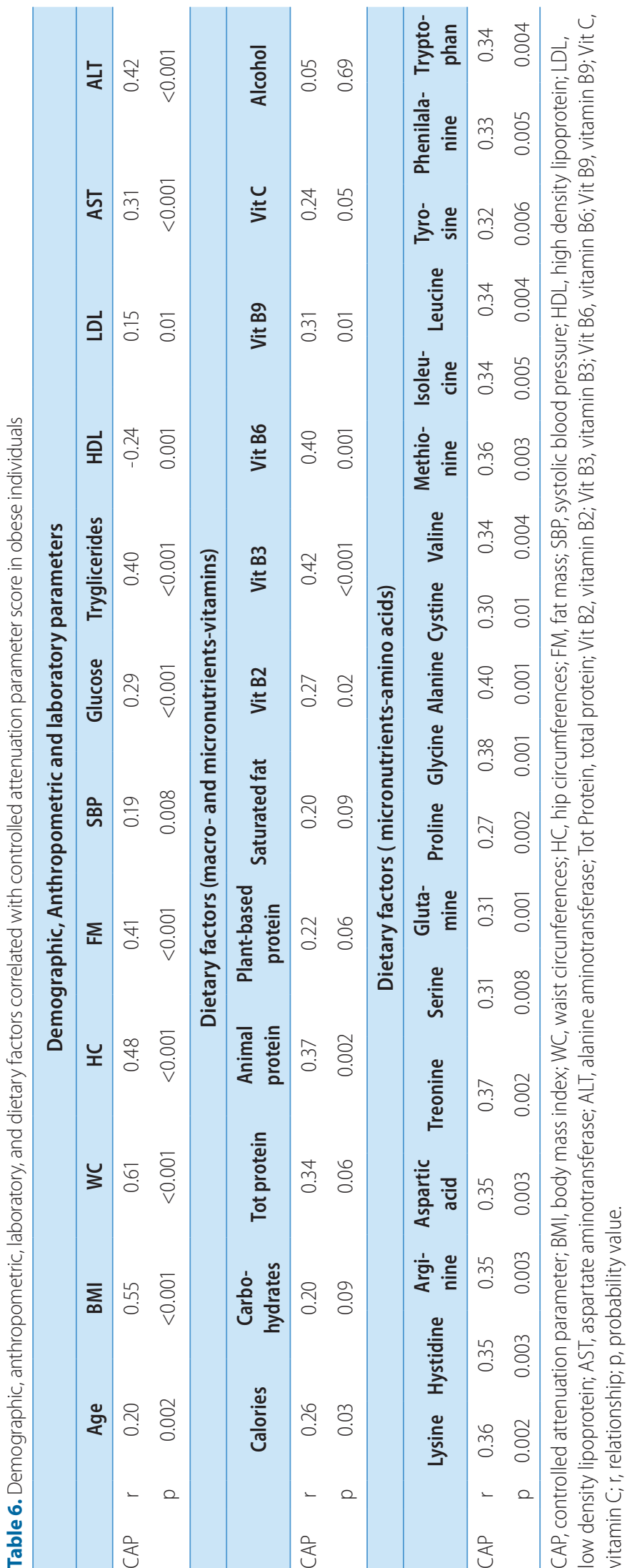

lated with the CAP score.

Table 3 shows the characteristics of the whole population according to vitamin B6 tertiles. Participants in tertile I (lowest vitamin B6 intake) had a lower CAP score than those in the tertile III (highest intake, $P=0.004$; post-hoc analysis, Table 3). All other factors were not significantly different between tertiles.

Logistic regression analysis (Table 4) showed that the tertiles of vitamin $\mathrm{B} 6$ intake were positively associated with the presence of hepatic steatosis $(B=-1.89 ; S E=0.85, P=0.026, C l$ 0.03-0.80) as well as tryglicerides, glucose, ALT and BMI. In the stepwise multivariable analysis, the CAP score remained still positively associated with vitamin $B 6$ tertiles $(B=8.14$; $\mathrm{SE}=4 ; P=0.04 ; \mathrm{Cl} 0.01-16.1$ ), tryglicerides, glucose, $\mathrm{ALT}$ and BMI while all other factors showed no association (Table 5).

\section{Subgroup with obesity}

The mean age was $51 \pm 11$ years. In this group, $89 \%$ had a CAP value $\geq 216,70 \% \geq 250$ and $36 \% \geq 300 \mathrm{dBm}-1$. Table 6 shows the factors that were correlated with CAP score. Again Vitamin B6 correlated with the CAP score $(r=0.40$; $P=0.01)$. As in the whole population, BMI, WC, glucose, triglycerides, ALT and AST again correlated with the CAP score. Unlike the whole population, age correlated with the CAP score $(r=0.20, P=0.02)$. Furthermore, a correlation was found between vitamin B3 intake and the CAP score, showing a higher $r$ value with a stronger $P$-value than the intake of other macronutrients and micronutrients $(r=0.42, P<0.001)$.

Table 7 shows the participants' characteristics according to vitamin B6 tertiles. Participants in tertile I had a lower CAP score than those in tertile III ( $P=0.01$; post-hoc analysis, Table 7$)$ and those in tertile II had a lower CAP score than participants in tertile III ( $P=0.04$; post-hoc analysis; Table 7). Participants in I tertile had also a lower SBP and DBP than those in tertile III ( $P=0.008$ and $P=0.001$ respectively; post-hoc analysis, Table 7) and those in tertile II had a lower SBP and DBP than individuals in tertile III ( $P=0.007 ; P=0.005$ respectively, post-hoc analysis; Table 7). In the stepwise multivariable analysis, the CAP score was still remained positively associated with total protein intake ( $B=0.56 ; S E=0.23 ; P=0.019 ; C l 0.10-1.02$ ), tryglicerides and ALT while all the other factors showed no association (Table 8). In tertile II, $40 \%$ of individuals had Steatosis grade $\mathrm{S} 2$ and $23 \%$ had $S 3^{10}$. In those in tertile III, $45 \%$ had steatosis grade $\mathrm{S} 3$ and $27 \%$ had $\mathrm{S} 2$. 
Yvelise Ferro, et al.

Table 7. Obese participant demographic, anthropometric, and clinical characteristics according to vitamin B6 tertile of intake

\begin{tabular}{|c|c|c|c|c|c|}
\hline Variables & $\begin{array}{l}\text { I tertile }(n=19) \\
1.0 \pm 0.1 \mathrm{mg} / \text { day }\end{array}$ & $\begin{array}{l}\text { Il tertile }(\mathrm{n}=19) \\
1.4 \pm 0.1 \mathrm{mg} / \text { day }\end{array}$ & $\begin{array}{l}\text { III tertile }(n=20) \\
2.1 \pm 0.3 \mathrm{mg} / \text { day }\end{array}$ & $P$-value & $\begin{array}{c}P \text {-value } \\
\text { Post-Hoc Analysis }\end{array}$ \\
\hline Age (years) & $52 \pm 9$ & $50 \pm 13$ & $51 \pm 13$ & 0.84 & NS \\
\hline BMI $\left(\mathrm{kg} / \mathrm{m}^{2}\right)$ & $36 \pm 6$ & $36 \pm 5$ & $36 \pm 5$ & 0.96 & NS \\
\hline WC (cm) & $113 \pm 15$ & $110 \pm 10$ & $112 \pm 13$ & 0.60 & NS \\
\hline $\mathrm{HC}(\mathrm{cm})$ & $117 \pm 14$ & $121 \pm 12$ & $119 \pm 11$ & 0.59 & NS \\
\hline $\mathrm{SBP}(\mathrm{mmHg})$ & $127 \pm 10$ & $127 \pm 10$ & $137 \pm 15$ & 0.010 & $\begin{array}{l}\text { | vs. ||| } 0.008 \\
\text { || vs. ||| } 0.007\end{array}$ \\
\hline $\mathrm{DBP}(\mathrm{mmHg})$ & $80 \pm 7$ & $79 \pm 7$ & $86 \pm 11$ & 0.012 & $\begin{array}{l}\text { I vs. ||| } 0.001 \\
\text { || vs. ||| } 0.005\end{array}$ \\
\hline FFM (\%) & $62 \pm 7$ & $59 \pm 7$ & $64 \pm 8$ & 0.18 & NS \\
\hline FM (\%) & $38 \pm 7$ & $40 \pm 7$ & $36 \pm 8$ & 0.18 & NS \\
\hline CAP score $(\mathrm{dB} / \mathrm{m})$ & $263 \pm 46$ & $268 \pm 40$ & $297 \pm 47$ & 0.038 & $\begin{array}{l}\text { | vs. ||| } 0.017 \\
\text { || vs. ||| } 0.042\end{array}$ \\
\hline Liver Stiffness (kPa) & $8.0 \pm 10$ & $5.5 \pm 1.5$ & $5.7 \pm 1.3$ & 0.36 & NS \\
\hline Glucose (mg/dL) & $97 \pm 14$ & $91 \pm 11$ & $98 \pm 11$ & 0.21 & NS \\
\hline Total cholesterol (mg/dL) & $196 \pm 42$ & $198 \pm 35$ & $198 \pm 34$ & 0.98 & NS \\
\hline HDL cholesterol (mg/dL) & $54 \pm 15$ & $56 \pm 10$ & $52 \pm 24$ & 0.83 & NS \\
\hline LDL cholesterol (mg/dL) & $114 \pm 33$ & $116 \pm 30$ & $112 \pm 39$ & 0.92 & NS \\
\hline Triglycerides (mg/dL) & $116 \pm 64$ & $121 \pm 50$ & $159 \pm 122$ & 0.21 & NS \\
\hline AST (IU/L) & $20 \pm 8$ & $22 \pm 6$ & $22 \pm 9$ & 0.42 & NS \\
\hline ALT (IU/L) & $23 \pm 14$ & $22 \pm 10$ & $27 \pm 19$ & 0.86 & NS \\
\hline \multicolumn{6}{|l|}{ Prevalence } \\
\hline Hyperlipidemia (\%) & 40 & 36 & 44 & 0.77 & \\
\hline Hypertension (\%) & 41 & 33 & 43 & 0.90 & \\
\hline Smokers (\%) & 41 & 29 & 48 & 0.67 & \\
\hline
\end{tabular}

BMI, body mass index; WC, waist circumferences; HC, hip circumferences; SBP, systolic blood pressure; DBP, diastolic blood pressure; FFM, free fat mass; FM, fat mass; CAP, controlled attenuation parameter; HDL, high density lipoprotein; LDL, low density lipoprotein; AST, aspartate aminotransferase; ALT, alanine aminotransferase; NS, non significant.

Table 8. Step-wise, multivariable linear regression analysis of factors associated with controlled attenuation parameter score in obese individuals

\begin{tabular}{lcccccc}
\hline $\begin{array}{l}\text { Dependent variable } \\
\text { (CAP score) }\end{array}$ & B & SE & $\boldsymbol{B}$ & $\mathbf{p}$ & \multicolumn{2}{c}{ C.I. 95\% } \\
\hline ALT & 1.17 & 0.41 & 0.46 & $<0.001$ & LL & UL \\
\hline Triglycerides & 0.19 & 0.06 & 0.35 & 0.003 & 0.07 & 2.52 \\
\hline Total protein intake & 0.56 & 0.23 & 0.28 & 0.019 & 0.10 & 0.32 \\
\hline
\end{tabular}

CAP, controlled attenuation parameter; B, unstandardized coefficients; SE, standard errors; $\beta$, standardized coefficients; $p$, probability value; C.I., confidence interval; LL, lower limit; UL, upper limit; ALT, alanine aminotransferase.

A Pearson's correlation identified foods groups correlated with vitamin $B 6$ intake confirming the lack of correlation between vitamin $B 6$ and alcohol or beer consumption $(P=0.39, r=0.11$ and $P=0.27, r=0.15$ respectively) while a correlation between intake of vitamin $\mathrm{B} 6$ and grains, meat and cheeses consumption and calories was present $(P=0.03, \mathrm{r}=0.29 ; P<0.001, \mathrm{r}=0.62, P=0.04$, $\mathrm{r}=0.38$ and $P<0.001, \mathrm{r}=0.48$, respectively; table not shown).

\section{DISCUSSION}

To the best of our knowledge, this is the first study demonstrat- 
ing the positive association between the presence of hepatic steatosis, assessed by $T E$, and tertiles of vitamin B6 intake. Furthermore, in obese individuals we found a positive association between the liver fat content, measured by the CAP score, and total protein intake. In this subgroup we found a correlation between the intake of vitamin B6 and food groups such as meat, grains, and cheeses. As expected, both the liver steatosis and CAP score were associated with serum glucose, triglycerides, BMI and ALT. Finally, we found a parallel increase in the CAP score with increasing vitamin B6 intake. In both the whole population and the subgroup of obese individuals, we found that participants in the lowest vitamin B6 tertile had a lower CAP score than those in the highest tertiles and all other variables showed no significant differences between tertiles.

Previous studies have demonstrated the detrimental effects that several dietary components have on promoting the development and progression of NAFLD. ${ }^{1,2}$ Although previous studies have attempted to establish the dietary risk factors for NAFLD in the general population using the 7-day food record, ${ }^{13,19}$ the role of vitamin B6 intake on liver steatosis has been scarcely studied in humans. There have also been few investigations into the association between body composition parameters and the CAP score. Nevertheless our study was not designed to explore the mechanisms underlying the pathogenic role of vitamin B6 on NAFLD. Instead it suggests that an association may exist, thus generating hypotheses for future research.

It has been already demonstrated that the synthesis of $A A$ from linoleate involves vitamin $B 6 .{ }^{20,21} A$ high production of $A A$ can increase the synthesis of $A A$-derived eicosanoids, curtailing that of eicosapentaenoic acid (EPA). ${ }^{22}$ This is now considered an important mechanism in the pathogenesis of NAFLD. ${ }^{23}$ In fact, high AA-derived eicosanoids lead to the synthesis of 2-series prostaglandins and thromboxanes and 4-series leukotrienes, which play a crucial role in regulating the production of proinflammatory cytokines, the production of reactive oxygen species and neutrophil activation. $^{23,24}$

Our study appear to diverge from other investigations which have shown a beneficial effect of dietary pyridoxal $5^{\prime}$-phosphate and pyridoxal, in particular on lipid glycation and lipid peroxidation. ${ }^{25}$ However, these positive effects were demonstrated in streptozotocin (STZ)-induced diabetic rats while no effect was found in normoglycaemic rats. ${ }^{25}$ In human studies, the clinical value of pyridoxine and pyridoxine-pyrrolidone carboxylate on liver disease has only been demonstrated in the treatment of acute alcohol intoxication, ${ }^{26}$ and with controversial results. ${ }^{27}$ It is clear that further research on this issue is needed to fully elucidate the precise role played by dietary vitamin B6 in the development of liver inflammation and steatosis.

In this study, a correlation was found between vitamin B3 intake and the CAP score, showing a higher $r$ value with a stronger $P$-value than the intake of other macronutrients and micronutrients $(r=0.42, P<0.001)$. However, we investigated the potential effects of vitamin $\mathrm{B} 6$ intake since it participates as a cofactor in transamination reactions, non-oxidative deamination and long chain fatty acid synthesis in the liver and needs to be obtained exogenously. ${ }^{7,8}$ On the contrary, the intestine is capable of partially synthesizing vitamin B3. Furthermore, dietary tryptophan can be converted into vitamin B3. The synthesis of vitamin B3 (niacin) from tryptophan depends on enzymes requiring vitamin B6. Thus, in our study we focused on vitamin B6 and excluded vitamin B3 from further statistical analyses because it is difficult to investigate the potential effects derived only from vitamin B3 contained in foods.

In our study, the positive association between liver steatosis and vitamin B6 may merely indicate a high meat consumption in those participants with a high CAP score. This is confirmed by the correlation between the CAP score and animal-based protein. Furthermore, the consumption of meat significantly contributes to vitamin B6 levels. ${ }^{28}$ The recommended daily intake of vitamin B6, for adults, is $1.7 \mathrm{mg}$ in men and $1.3 \mathrm{mg}$ in women ${ }^{29}$ while in our investigation, participants from tertile III (50\% were male) exceeded these levels. It has already been demonstrated that over-consumption of meat and grains increases the risk of developing NAFLD. ${ }^{30,31}$ Individuals with NAFLD have a greater intake of total protein per day compared to those without NAFLD. ${ }^{32}$ In line with these findings, our study confirms the importance of restricting the consumption of particular food groups, in order to also limit the amount of vitamin B6. Furthermore, in the subgroup of obese individuals, we found a correlation between the intake of vitamin B6 and meat, grains, and cheese consumption.

Some strengths and weaknesses must be pointed out in this study. In order to detect hepatic steatosis, three different cutoffs were evaluated. Currently, numerous research on CAP technology has been performed on small cohorts that differ in term of their etiologies and cut-offs. Consequently, in this study three cut-offs were chosen because they include most of the previously published cut-offs for the diagnosis of steatosis $>33 \%$. $^{10,11}$ However, irrespective of the steatosis grade and cutoffs used, an important finding in our study is an increase in 
the CAP score with an increasing vitamin B6 intake.In this study, we did not carry out a liver ultrasonography which is the most common liver imaging technique used in both research and clinical practice. However, it is well known that it is highly operator- and machine-dependant and cannot quantify steatosis. ${ }^{33}$ CAP assessment has overcome this limitation, with the additional advantage of being able to exclude fibrosis and cirrhosis. Furthermore, it is well known that a liver biopsy is the gold standard for the assessment of hepatic steatosis, necroinflammation and fibrosis and, in this regard, now the CAP score provides a standardized non-invasive measure of hepatic steatosis. ${ }^{34}$ Our study was limited by its cross-sectional design, thus, it is impossible to infer causality. In particular, it is difficult to prove that a 7-day intake of vitamin B6 could have an effect on liver steatosis development, which is a slow progressive disease. Furthermore, data collected in this manner may be open to errors due to the method of data acquisition as well as the unreliability of patient recall and/or reporting. However, previous studies have identified the dietary risk factors for NAFLD using this method. ${ }^{13,19}$ In addition, it is well accepted that a 7-day record gives a representative measure of the habitual dietary intake, especially when conducted under the same conditions. ${ }^{35-37}$ Several fundamental studies found an association between hepatic damage and increasing doses of alcohol, which were estimated only on the basis of a dietary intake assessment. ${ }^{13,38,39}$ Our study suggests the possibility that an increased vitamin B6/total protein intake may have a role in the pathogenesis of fatty liver.

Finally, we believe that this study raises several important issues regarding the independent influence of vitamin B6, BMI or other anthropometric and laboratory parameters on hepatic steatosis. However, despite it is being well accepted that only visceral obesity seems to be the main source of pro-steatogenic mediators, it also has been suggested that, among patients with hepatic steatosis, the presence of visceral obesity does not seem to be a major determinant for non-alcoholic steatohepatitis. ${ }^{40}$ In humans, genetic polymorphisms associated with NAFLD may also dissociate hepatic steatosis from obesity and insulin resistance. ${ }^{41}$ In addition, a percentage of NAFLD patients in tertiary care are lean and, compared with obese individuals, had higher aminotransferase levels. ${ }^{40}$ Finally, NAFLD is more frequently ignored in normal weight populations who may have a high total protein intake. ${ }^{40}$ Further studies, specifically in this set of patients, are necessary in order to better understand the nature of the interaction between all these po- tential risk factors for hepatic steatosis. We believe that these results can help to suggest other mechanisms and to hypothesize new dietary factors involved in the pathogenesis of NAFLD.

\section{Authors' contribution}

TM and AP were responsible for study design, data analysis, manuscript writing. YF, IC and CT were responsible for enrollment and statistical analysis. EM and FP were responsible for anthropometric and nutritional measurements. SR and CC revised nutritional data and manuscript. All authors approved final manuscript.

\section{Conflicts of Interest}

The authors have no conflicts to disclose.

\section{REFERENCES}

1. Ekstedt M, Franzén LE, Mathiesen UL, Thorelius L, Holmqvist M, Bodemar $\mathrm{G}$, et al. Long-term follow-up of patients with NAFLD and elevated liver enzymes. Hepatology 2006;44:865-873.

2. Younossi ZM, Koenig AB, Abdelatif D, Fazel Y, Henry L, Wymer M. Global epidemiology of nonalcoholic fatty liver disease-Meta-analytic assessment of prevalence, incidence and outcomes. Hepatology 2016;64:73-84.

3. Maciejewska D, Ossowski P, Drozd A, Ryterska K, Jamioł-Milc D, Banaszczak $M$, et al. Metabolites of arachidonic acid and linoleic acid in early stages of non-alcoholic fatty liver disease--A pilot study. Prostaglandins Other Lipid Mediat 2015;121(Pt B):184-189.

4. Rossaint J, Nadler JL, Ley K, Zarbock A. Eliminating or blocking 12/15-lipoxygenase reduces neutrophil recruitment in mouse models of acute lung injury. Crit Care 2012;16:R166.

5. Vonakis BM, Vanderhoek JY. 15-Hydroxyeicosatetraenoic acid (15HETE) receptors. Involvement in the 15-HETE-induced stimulation of the cryptic 5-lipoxygenase in PT-18 mast/basophil cells. J Biol Chem 1992;267: 23625-23631.

6. Cunnane SC, Manku MS, Horrobin DF. Accumulation of linoleic and gamma-linolenic acids in tissue lipids of pyridoxine-deficient rats. J Nutr 1984;114:1754-1761.

7. Dussault PE, Lepage M. Effects of pyridoxine deficiency on the composition of plasma and liver fatty acids in rats fed low and high fat diets. J Nutr 1975;105:1371-1376.

8. Saareks V, Mucha I, Sievi E, Riutta A. Nicotinic acid and pyridoxine modulate arachidonic acid metabolism in vitro and ex vivo in man. Pharmacol Toxicol 1999;84:274-280.

9. Sasso $M$, Beaugrand $M$, de Ledinghen $V$, Douvin $C$, Marcellin $P_{1}$ Poupon $\mathrm{R}$, et al. Controlled attenuation parameter (CAP): a novel 
VCTE(TM) guided ultrasonic attenuation measurement for the evaluation of hepatic steatosis: preliminary study and validation in a cohort of patients with chronic liver disease from various causes. Ultrasound Med Biol 2010;36:1825-1835.

10. de Lédinghen V, Vergniol J, Foucher J, Merrouche W, le Bail B. Noninvasive diagnosis of liver steatosis using controlled attenuation parameter (CAP) and transient elastography. Liver Int 2012;32:911-918.

11. Myers RP, Pollett A, Kirsch R, Pomier-Layrargues G, Beaton M, Levstik $M$, et al. Controlled Attenuation Parameter (CAP): a non-invasive method for the detection of hepatic steatosis based on transient elastography. Liver Int 2012;32:902-910.

12. Pujia A, Gazzaruso C, Ferro Y, Mazza E, Maurotti S, Russo C, et al. Individuals with metabolically healthy overweight/obesity have higher fat utilization than metabolically unhealthy individuals. Nutrients 2016;8. pii: E2. doi: 10.3390/nu8010002.

13. Bedogni G, Miglioli L, Masutti F, Tiribelli C, Marchesini G, Bellentani S. Prevalence of and risk factors for nonalcoholic fatty liver disease: the Dionysos nutrition and liver study. Hepatology 2005;42:44-52.

14. Centers for Disease Control and Prevention. Tobacco use among adults - United States, 2005. Atlanta: MMWR, 2006, 55: 11451148.

15. Psaty BM, Furberg CD, Kuller LH, Bild DE, Rautaharju PM, Polak JF, et al. Traditional risk factors and subclinical disease measures as predictors of first myocardial infarction in older adults: the Cardiovascular Health Study. Arch Intern Med 1999;59:1339-1347.

16. Montalcini T, Gorgone G, Federico D, Ceravolo R, Emanuele V, Sesti $\mathrm{G}$, et al. Association of LDL cholesterol with carotid atherosclerosis in menopausal women affected by the metabolic syndrome. Nutr Metab Cardiovasc Dis 2005;15:368-372.

17. Montalcini T, Gorgone G, Garzaniti A, Gazzaruso C, Pujia A. Artery remodeling and abdominal adiposity in nonobese postmenopausal women. Eur J Clin Nutr 2010;64:1022-1024.

18. Talluri T, Lietdke RJ, Evangelisti A, Talluri J, Maggia G. Fat-free mass qualitative assessment with bioelectric impedance analysis (BIA). Ann N Y Acad Sci 1999;873:94-98.

19. Ouyang X, Cirillo P, Sautin Y, McCall S, Bruchette JL, Diehl AM, et al. Fructose consumption as a risk factor for non-alcoholic fatty liver disease. J Hepatol 2008;48:993-999.

20. She QB, Hayakawa T, Tsuge H. Effect of vitamin B6 deficiency on linoleic acid desaturation in the arachidonic acid biosynthesis of rat liver microsomes. Biosci Biotechnol Biochem 1994;58:459-463.

21. An BK, Tanaka K, Ohtani S. Effects of dietary vitamin B 6 levels on lipid concentration and fatty acid composition in growing chicks. Asian-Australasian Journal of Animal Sciences 1995,8:627-634.

22. Molendi-Coste O, Legry V, Leclercq IA. Why and how meet n-3 PUFA dietary recommendations? Gastroenterol Res Pract 2010;2011:364040.

23. Scorletti E, Byrne CD. Omega-3 fatty acids, hepatic lipid metabolism, and nonalcoholic fatty liver disease. Annu Rev Nutr 2013;33:231-248.

24. Cubero FJ, Nieto N. Arachidonic acid stimulates TNF $\alpha$ production in Kupffer cells via a reactive oxygen species-pERK1/2-Egr1-dependent mechanism. Am J Physiol Gastrointest Liver Physiol 2012;303:G228 G239.

25. Higuchi O, Nakagawa K, Tsuzuki T, Suzuki T, Oikawa S, Miyazawa T. Aminophospholipid glycation and its inhibitor screening system: a new role of pyridoxal 5 '-phosphate as the inhibitor. J Lipid Res 2006;47:964-974.

26. Caballería J, Parés A, Brú C, Mercader J, Plaza AG, Caballería L, et al. Metadoxine accelerates fatty liver recovery in alcoholic patients: results of a randomized double-blind, placebo-control trial. J Hepatol 1998;28:54-60

27. Lheureux P, Penaloza A, Gris M. Pyridoxine in clinical toxicology: a review. Eur J Emerg Med 2005;12:78-85.

28. Zelber-Sagi S, Ratziu V, Oren R. Nutrition and physical activity in NAFLD: An overview of the epidemiological evidence. World J Gastroenterol 2011;17:3377-3389.

29. Institute of Medicine (US) Standing Committee on the Scientific Evaluation of Dietary Reference Intakes. Dietary Reference Intakes for Thiamin, Riboflavin, Niacin, Vitamin B6, Folate, Vitamin B12, Pantothenic acid, Biotin and Choline. Washington DC: National Academy Press, 1998: 150-188.

30. Zelber-Sagi S, Nitzan-Kaluski D, Goldsmith R, Webb M, Blendis L, Halpern Z, et al. Long term nutritional intake and the risk for nonalcoholic fatty liver disease (NAFLD): a population based study. J Hepatol 2007;47:711-717.

31. Zanovec M, O'Neil CE, Keast DR, Fulgoni VL 3rd, Nicklas TA. Lean beef contributes significant amounts of key nutrients to the diets of US adults: National Health and Nutrition Examination Survey 19992004. Nutr Res 2010;30:375-381.

32. Chan R, Wong VW, Chu WC, Wong GL, Li LS, Leung J, et al. Higher estimated net endogenous Acid production may be associated with increased prevalence of nonalcoholic Fatty liver disease in Chinese adults in Hong Kong. PLoS One 2015;10:e0122406.

33. Castera L, Vilgrain V, Angulo P. Noninvasive evaluation of NAFLD. Nat Rev Gastroenterol Hepatol 2013;10:666-675.

34. de Lédinghen V, Wong GL, Vergniol J, Chan HL, Hiriart JB, Chan AW, et al. Controlled attenuation parameter for the diagnosis of steatosis in non-alcoholic fatty liver disease. J Gastroenterol Hepatol 2016;31:848-855.

35. .Høidrup S, Andreasen AH, Osler M, Pedersen AN, Jørgensen LM, Jørgensen $T$, et al. Assessment of habitual energy and macronutrient intake in adults: comparison of a seven day food record with a dietary history interview. Eur J Clin Nutr 2002;56:105-113.

36. Bingham SA. Limitations of the various methods for collecting dietary intake data. Ann Nutr Metab 1991;35:117-127.

37. Willett WC. Nutritional Epidemiology. 2nd ed. New York: Oxford 
Yvelise Ferro, et al.

Liver and vitamin $\mathrm{B} 6$

University Press, 1998:110-124.

38. Hassan MM, Hwang LY, Hatten CJ, Swaim M, Li D, Abbruzzese JL, et al. Risk factors for hepatocellular carcinoma: synergism of alcohol with viral hepatitis and diabetes mellitus. Hepatology 2002;36:12061213.

39. Donato F, Tagger A, Gelatti U, Parrinello G, Boffetta P, Albertini A, et al. Alcohol and hepatocellular carcinoma: the effect of lifetime intake and hepatitis virus infections in men and women. Am J Epi- demiol 2002;155:323-331.

40. Verdelho Machado M, Cortez-Pinto H. Fatty liver in lean patients: is it a different disease? Ann Gastroenterol 2012;25:1-2.

41. Mondul A, Mancina RM, Merlo A, Dongiovanni P, Rametta R, Montalcini T, et al. PNPLA3 I148M Variant Influences Circulating Retinol in Adults with Nonalcoholic Fatty Liver Disease or Obesity. J Nutr 2015;145:1687-1691 